\title{
A PARTITION THEOREM FOR TRIPLES ${ }^{1}$
}

\author{
E. C. MILNER AND K. PRIKRY
}

\begin{abstract}
Consider a partition of triples of enumerable ordinals into two classes. We show that either for each natural number $k$, the first class contains all triples from a set of type $\omega+k$, or the second class contains all triples of a four element set.
\end{abstract}

1. Introduction. If $\gamma$ is an ordinal, $r$ a positive integer and $\varphi, \psi_{i}(i<\gamma)$ linear order types, then the partition symbol

$$
\varphi \rightarrow\left(\psi_{i}\right)_{i<\gamma}^{r}
$$

of Erdős and Rado [5] means that the following statement is true: whenever $(S,<)$ is a linearly ordered set of type $\varphi$ and $[S]^{r}=\{X \subseteq S:|X|=r\}$ is partitioned into $\gamma$ classes $K_{i}(i<\gamma)$, then there are $i<\gamma$ and $B \subset S$ such that $(B,<)$ has order type $\psi_{i}$ and $B$ is homogeneous for the class $K_{i}$, i.e. $[B]^{r} \subseteq K_{i}$. The negation of (1.1) is written $\varphi \nrightarrow\left(\psi_{i}\right)_{i<\gamma}^{r}$. Also, when $\gamma=2$ we write $(1.1)$ as $\varphi \rightarrow\left(\psi_{0}, \psi_{1}\right)^{r}$.

Partition relations have played a central role in the study of many set-theoretic problems, and a great deal is now known about such relations especially for the case when the order types are initial ordinals, i.e. cardinal numbers (see [4]). Rather less is known when the order types are not cardinals and most of the known results of this kind are for the case $r=2$. The only relations for order types (as opposed to cardinals) that we know for $r \geq 3$ are the following:

$$
\begin{aligned}
& \omega_{1} \nrightarrow(\omega+2, r+1)^{r} \quad \text { and } \lambda \nrightarrow(\omega+2, r+1)^{r} \quad(r \geq 4), \\
& \lambda \nrightarrow(\omega+2, \omega)^{3}, \\
& \omega_{1} \nrightarrow(\omega+2, \omega)^{3}, \\
& \psi \rightarrow(\omega+k, 4)^{3} \quad(k<\omega), \\
& \varphi \rightarrow(\omega+1)_{s}^{r} \quad(r, s<\omega) .
\end{aligned}
$$

Here $\lambda$ denotes the order type of the reals with the usual ordering, $\psi$ is any real type, i.e. an uncountable type such that $\psi \nsupseteq \omega_{1}$ and $\psi \nsupseteq \omega_{1}^{*}$, and $\varphi$ denotes any linear order satisfying the relation

$$
\varphi \rightarrow(\omega)_{\omega}^{1}
$$

(in other words $\varphi$ is not a countable union of reverse well ordered types). All the above results were proved by Erdős and Rado [5] except for (1.6). They only proved (1.6) for the special case $\varphi=\omega_{1}$, and Galvin [6] gave a proof which works

Received by the editors September 18, 1984.

1980 Mathematics Subject Classification. Primary 03E05, 03E50, 04A20, 06A05.

Key words and phrases. Partition relation, Martin's Axiom, real type.

${ }^{1}$ Research supported by NSERC Grant No. A5198 and NSF Grant MCS 830361. 
for arbitrary $\varphi$ satisfying (1.7). In this paper we prove the following companion of (1.5),

$$
\omega_{1} \rightarrow(\omega+k, 4)^{3} \quad(k<\omega) .
$$

The proof of (1.5) given by Erdős and Rado [5, Theorem 31] is quite complicated and relies in an essential way upon the fact that any real type $\psi$ is the ordinal sum $\psi=\psi_{0}+\psi_{1}$ of two real types $\psi_{0}, \psi_{1}$. It is for this reason that their method of proof did not yield (1.8).

We shall prove the relation (1.8) under the additional assumption that Martin's axiom $\mathrm{MA}_{\omega_{1}}$, is true. It then follows, by an argument due to Baumgartner and Hajnal [2, Theorems 3 and 4], that, since (1.8) is an absolute statement relative to a countable chain condition extension, it is also a theorem of ZFC.

As frequently happens, when corresponding partition relations like (1.5) and (1.8) hold for real types and for $\omega_{1}$, then the same relation holds for the wider class of order types $\varphi$ satisfying (1.7). This is the case here. In $\S 4$ we indicate briefly how our proof of (1.8) may be modified to prove that

$$
\varphi \rightarrow(\omega+k, 4)^{3} \quad(k<\omega)
$$

holds for any $\varphi$ satisfying (1.7). Again, this resembles the Baumgartner and Hajnal proof [2] that

$$
\varphi \rightarrow(\alpha)_{k}^{2} \quad\left(\alpha<\omega_{1}, k<\omega\right) .
$$

No doubt a direct proof of (1.9) can be given along the same lines that Galvin [7] used to prove (1.10), but we did not investigate this.

It is natural to conjecture that (1.9) can be strengthened to

$$
\varphi \rightarrow(\alpha, n)^{3} \quad\left(\alpha<\omega_{1}, n<\omega\right),
$$

but we were unable to prove either of the following "simplest cases" not covered by (1.9):

$$
(?) \omega_{1} \rightarrow(\omega \cdot 2,4)^{3}, \quad(?) \omega_{1} \rightarrow(\omega+2,5)^{3} .
$$

Another interesting question is whether $\lambda$ can be replaced by any real type in (1.3).

2. Notation. We use standard set theoretic notation as used, for example, in Jech [8].

If $(S,<)$ is a linearly ordered set and $x \in S$, then we write $S(\geq x)$ to denote the set $\{y \in S: y \geq x\}$. Also, if $X$ and $Y$ are subsets of $S$, we write $X<Y$ to indicate that $x<y$ holds for all $x \in X$ and $y \in Y$.

Let $(S,<)$ be an ordered set and let $[S]^{r}=\{X \subseteq S:|X|=r\}=\bigcup\left\{K_{i}: i<\gamma\right\}$ be any partition. Then for an order type $\psi$, we write $\psi \in\left[K_{i}\right]$ if there is a subset $B \subseteq S$ such that $(B,<)$ has order type $\operatorname{tp}(B,<)=\psi$ and $B$ is homogeneous for the class $K_{i}$, i.e. $[B]^{r} \subseteq K_{i}$. For $r, s<\omega$ and $A, B \subseteq S$ we write $[A]^{r} \otimes[B]^{s}$ to denote the set $\{X \subseteq A \cup B:|X \cap A|=r,|X \cap B|=s\}$.

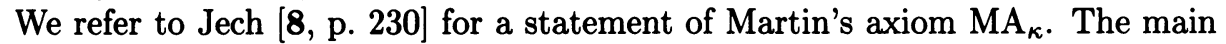
use that we make of this axiom is contained in the following two lemmas; Lemma 2.2 is an easy consequence of Lemma 2.1 and Ramsey's theorem. For Lemma 2.1, see e.g. [9, Theorem 7]. 
LEMMA 2.1. Assume $\mathrm{MA}_{\lambda}$. Let $A_{\rho} \in[\omega]^{\omega}(\rho \in \lambda)$ and any finitely many of these sets have infinite intersection. Then there is a set $X \in[\omega]^{\omega}$ such that $X \backslash A_{\rho}$ is finite for all $\rho<\lambda$.

LEMMA 2.2. Assume $\mathrm{MA}_{\lambda}$ holds for $\lambda<\kappa$. Suppose that, for each $\rho<\kappa$, $r_{\rho}, k_{\rho}<\omega$ and $f_{\rho}:[\omega]^{r_{\rho}} \rightarrow k_{\rho}$. Then there is an ultrafilter $\mathcal{U}$ on $\omega$ such that for each $\rho<\kappa$ there is an $f_{\rho}$-homogeneous set $A_{\rho} \in \mathcal{U}$ (i.e. $f_{\rho} \uparrow\left[A_{\rho}\right]^{r_{\rho}}$ is constant).

3. A proof of (1.8) assuming $\mathrm{MA}_{\omega_{1}}$. In this section the letters $A$ and $B$ (possibly with suffixes or primes) will always denote subsets of $\omega_{1}$ which respectively have order types $\omega$ and $\omega_{1}$.

Let $\left[\omega_{1}\right]^{3}=K_{0} \cup K_{1}$. We want to show that either

$$
\omega+k \in\left[K_{0}\right]
$$

or

$$
4 \in\left[K_{1}\right] .
$$

LEMMA 3.1. Assume $\mathrm{MA}_{\omega_{1}}, A \subseteq \omega_{1}, B \subseteq \omega_{1}$. Then there are $i<2, A^{\prime} \subseteq A$, $B^{\prime} \subseteq B$ such that $\left[A^{\prime}\right]^{2} \otimes\left[B^{\prime}\right]^{1} \subseteq K_{i}$.

PROOF. By Lemma 2.2 (MA is not needed for this case) there is an ultrafilter $\mathcal{U}$ on $A$ such that for each $\rho \in B$ there are $\tilde{A}_{\rho} \in \mathcal{U}$ and $i(\rho)<2$ such that $\left[\tilde{A}_{\rho}\right]^{2} \otimes[\{\rho\}] \subseteq K_{i(\rho)}$. By Lemma 2.1 there is $A^{*} \subseteq A$ such that $A^{*} \backslash \tilde{A}_{\rho}$ is finite for all $\rho \in B$. Since $B$ is uncountable, there are $i<2, a \in A^{*}$ and $B^{\prime} \subseteq B$ such that $A^{\prime}=A^{*}(\geq a) \subseteq \tilde{A}_{\rho}$ and $i(\rho)=i$ for all $\rho \in B^{\prime}$. Then $\left[A^{\prime}\right]^{2} \otimes\left[B^{\prime}\right]^{1} \subseteq K_{i}$.

LEMMA 3.2. Assume $\mathrm{MA}_{\omega_{1}}$. If $B \subseteq \omega_{1}$ is such that

$$
\left[A^{\prime}\right]^{2} \otimes\left[B^{\prime}\right]^{1} \nsubseteq K_{0}
$$

for all $A^{\prime}, B^{\prime} \subseteq B$, then there are sets $A_{n} \subseteq B(n \leq \omega)$ such that $A_{m}<A_{n}$ and $\left[A_{m}\right]^{2} \otimes\left[A_{n}\right]^{1} \subseteq K_{1}$ for $m<n \leq \omega$.

ProOF. By (3.3) and repeated applications of Lemma 3.1, it follows that there are sets $A_{n}^{\prime}, B_{n}^{\prime} \subseteq B(n<\omega)$ such that $A_{n}^{\prime}<B_{n}^{\prime}, A_{n+1}^{\prime} \cup B_{n+1}^{\prime} \subseteq B_{n}$ and $\left[A_{n}^{\prime}\right]^{2} \otimes\left[B_{n}^{\prime}\right]^{1} \subseteq K_{1}$.

By Lemma 2.2 there is an ultrafilter $\mathcal{U}_{n}$ on $A_{n}^{\prime}$ such that, for each $\rho \in B$, there are $\tilde{A}_{n, \rho} \in \mathcal{U}_{n}$ and $i(n, \rho)<2$ such that $\left[\tilde{A}_{n, \rho}\right]^{2} \otimes[\{\rho\}]^{1} \subseteq K_{i(n, \rho)}$.

Suppose that for some $n<\omega$ there are uncountably many $\rho \in B$ such that $i(n, \rho)=0$. Applying Lemma 2.1 as in the second half of the proof of Lemma 3.1, we find $A^{\prime} \subseteq A_{n}^{\prime}$ and $B^{\prime} \subseteq B$ such that $\left[A^{\prime}\right]^{2} \otimes\left[B^{\prime}\right]^{1} \subseteq K_{0}$, which contradicts (3.3). Without loss of generality we may therefore assume that $i(n, \rho)=1$ for all $n<\omega$ and $\rho \in B$.

Let $n<\omega$ and suppose that we have already chosen elements $a_{j} \in B$ and sets $A_{j} \subseteq A_{j}^{\prime}$ for $j<n$ and a set $B_{n} \subseteq B$ such that

$$
\bigcup_{j<\omega} A_{j}^{\prime}<\left\{a_{0}\right\}<\left\{a_{1}\right\}<\cdots<\left\{a_{n-1}\right\}<B_{n}
$$

and

$$
A_{j} \subseteq \tilde{A}_{j, \rho} \text { if } j<n \text { and } \rho \in B_{n} \cup\left\{a_{0}, \ldots, a_{n-1}\right\}
$$


By the same argument as used in the proof of Lemma 3.1, there are $A_{n} \subseteq$ $\bigcap_{j<n} \tilde{A}_{n, a_{j}}$ and $B_{n+1}^{*} \subseteq B_{n}$ such that $A_{n} \subseteq \tilde{A}_{n, \rho}$ for all $\rho \in B_{n+1}^{*}$. Choose any element $a_{n} \in B_{n+1}^{*}$ and set $B_{n+1}=\left\{x \in B_{n+1}^{*}: x>a_{n}\right\}$. Then the sets $A_{n}(n<\omega)$ and $A_{\omega}=\left\{a_{j}: j<\omega\right\}$ satisfy the requirements of the lemma.

We now prove (1.8) under the assumption that $\mathrm{MA}_{\omega_{1}}$ holds. As we have already remarked in $\S 1$, it follows by an absoluteness argument of Baumgartner and Hajnal that (1.8) is also a theorem of ZFC.

Case 1. There are $A_{0}, B_{0} \subseteq \omega_{1}$ such that $\left[A_{0}\right]^{2} \otimes\left[B_{0}\right]^{1} \subseteq K_{0}$. We may assume that $A_{0}<B_{0}$. Let $U$ be an ultrafilter on $A_{0}$. For each set $X \in\left[B_{0}\right]^{2}$, there is $i(X)<2$ such that $A(X)=\left\{a \in A_{0}:\{a\} \cup X \in K_{i(X)}\right\} \in \mathcal{U}$. By the DushnikMiller theorem [3], (see also [4, p. 70], $\omega_{1} \rightarrow\left(\omega, \omega_{1}\right)^{2}$, and so either (i) there is $A_{1} \subseteq B_{0}$ such that $i(X)=0\left(\forall X \in\left[A_{1}\right]^{2}\right)$, or (ii) there is $B_{1} \subseteq B_{0}$ such that $i(X)=1\left(\forall X \in\left[B_{1}\right]^{2}\right)$.

Suppose (i) holds. By Ramsey's theorem we may assume that $\left[A_{i}\right]^{3} \subseteq K_{0}(i<2)$, otherwise (3.2) holds. Choose $W \in\left[A_{1}\right]^{k}$ and let $A=\bigcap_{X \in[W]^{2}} A(X)$. Then $A \cup W$ has order type $\omega+k$ and $[A \cup W]^{3} \subseteq K_{0}$ so that (3.1) holds.

Suppose (ii) holds. If (3.1) is false, then there is $Z \in\left[B_{1}\right]^{3}$ such that $Z \in K_{1}$. Choose $z \in \bigcap\left\{A(X): X \in[Z]^{2}\right\}$. Then $[\{z\} \cup Z]^{3} \subseteq K_{1}$ and (3.2) holds.

Case 2. $[A]^{2} \otimes[B]^{1} \nsubseteq K_{0}$ for all $A, B \subseteq \omega_{1}$.

In this case, by Lemma 3.2, there are sets $A_{n} \subseteq \omega_{1}(n \leq \omega)$ such that $A_{0}<$ $A_{1}<\cdots<A_{\omega}$ and $\left[A_{m}\right]^{2} \otimes\left[A_{n}\right]^{1} \subseteq K_{1}$ for $m<n \leq \omega$. By Ramsey's theorem we may assume that $\left[A_{\omega}\right]^{3} \subseteq K_{0}$, otherwise (3.2) holds. Let $Y=\left\{y_{i}: i<k\right\} \in\left[A_{\omega}\right]^{k}$. We may assume that, for $X \in[Y]^{2}$ and $n<\omega$, there is at most one element $a \in A_{n}$ such that $\{a\} \cup X \in K_{1}$, otherwise (3.2) holds. Thus, without loss of generality we may suppose that $\left[\bigcup_{n<\omega} A_{n}\right]^{1} \otimes[Y]^{2} \subseteq K_{0}$.

Consider the partition

$$
\bigcup_{m<n<\omega}\left[A_{m}\right]^{1} \otimes\left[A_{n}\right]^{1}=J_{0} \cup J_{1} \cup \cdots \cup J_{k},
$$

in which $\left\{a, a^{\prime}\right\} \in J_{j}(j<k)$ if $j$ is the least integer such that $\left\{a, a^{\prime}, y_{j}\right\} \in K_{1}$, and $\left\{a, a^{\prime}\right\} \in J_{k}$ if $\left\{a, a^{\prime}, y_{j}\right\} \in K_{0}$ for all $j<k$. Suppose that

$$
\begin{aligned}
& \text { whenever } n_{0}<n_{1}<\cdots<\omega \text { and } A_{n_{i}}^{\prime} \subseteq A_{n_{i}}(i<\omega) \text {, then } \\
& \text { there are } a \in A_{n_{0}}^{\prime} \text { and indices } i(l)(l<\omega) \text { such that } \\
& 0<i(0)<i(1)<\cdots \text { and sets } A_{n_{i_{(l)}}}^{\prime \prime} \subseteq A_{n_{i(l)}}^{\prime} \text { such that } \\
& \left\{a, a^{\prime}\right\} \in J_{k} \text { for all } a^{\prime} \in \bigcup_{l<\omega} A_{n_{i_{(l)}}}^{\prime \prime} .
\end{aligned}
$$

Then we may inductively choose indices $n_{j}(j<\omega)$ and elements $a_{j} \in A_{n_{j}}(j<$ $\omega)$ so that all pairs $\left\{a_{j}, a_{j^{\prime}}\right\}$ belong to $J_{k}$ for $j<j^{\prime}<\omega$. Put $A=\left\{a_{j}: j<\omega\right\}$. As before, we may assume that $[A]^{3} \subseteq K_{0}$. Then $A \cup Y$ is a subset of $\omega_{1}$ of type $\omega+k$ and $[A \cup Y]^{3} \subseteq K_{0}$, i.e. (3.1) holds.

Therefore, we may assume that (3.4) is false. Without loss of generality we may therefore suppose that for each element $a \in A_{0}$ the set

$$
N(a)=\left\{n<\omega:\left\{a, a^{\prime}\right\} \in J_{k} \text { for infinitely many } a^{\prime} \in A_{n}\right\}
$$

is finite. Let $Z \in\left[A_{0}\right]^{k+1}$ and let $n \in \omega \backslash \bigcup_{a \in Z} N(a)$. Then there is some element $a^{\prime} \in A_{n}$ such that $\left\{a, a^{\prime}\right\} \notin J_{k}$ for all $a \in Z$. Hence, there are $a_{0}, a_{1} \in Z$ and $j<k$ such that $\left\{a_{0}, a^{\prime}\right\}$ and $\left\{a_{1}, a^{\prime}\right\}$ both belong to $J_{j}$. It follows that $\left[\left\{a_{0}, a_{1}, a^{\prime}, y_{j}\right\}\right]^{3} \in$ $K_{1}$, i.e. $4 \in\left[K_{1}\right]$. 
4. Types which satisfy $\varphi \rightarrow(\omega)_{\omega}^{1}$. Let $\Phi$ denote the class of all linear order types $\varphi$ which satisfy the partition relation $\varphi \rightarrow(\omega)_{\omega}^{1}$. For $\varphi \in \Phi$, we say that $\varphi$ is right-irreducible if $\varphi=\varphi_{0}+\varphi_{1}, \varphi_{1} \neq 0$ implies $\varphi_{1} \in \Phi$. In order to prove (1.3) for types $\varphi \in \Phi$ we need the following simple facts.

LEMMA 4.1. If $\varphi \in \Phi$, then $\varphi$ can be written as $\varphi=\varphi_{0}+\varphi_{1}$, where $\varphi_{0} \in \Phi$ and $\varphi_{0}$ is right-irreducible.

ProOF. Let $(S,<)$ be a linearly ordered set of type $\varphi$. Let $S_{1}=\{x \in S$ : $\operatorname{tp}(S(\geq x),<) \notin \Phi\}, S_{0}=S \backslash S_{1}, \varphi_{i}=\operatorname{tp}\left(S_{i},<\right)(i<2)$. Then $\varphi=\varphi_{0}+\varphi_{1}$. It will be enough to show that $\varphi_{1} \notin \Phi$. Let $\left\{x_{\rho}: \rho<\lambda\right\}$ be a coinitial sequence in $S_{1}$ such that $x_{0}>x_{1}>\cdots$. Then, for $\rho<\lambda$, there is an $\omega$-decomposition of $X_{\rho}=S\left(\geq x_{\rho}\right) \backslash \bigcup_{\sigma<\rho} S\left(\geq x_{\sigma}\right)$, say $X_{\rho}=\bigcup_{n<\omega} X_{\rho n}$, such that $\operatorname{tp}\left(X_{\rho n},<\right) \geq \omega$. Put $Y_{n}=\bigcup_{\rho<\lambda} X_{\rho n}$. Then $S_{1}=\bigcup_{n<\omega} Y_{n}$ and $\operatorname{tp}\left(Y_{n},<\right) \geq \omega$ since $X_{0}>X_{1}>\cdots$.

LEMMA 4.2. If $\varphi \in \Phi$, then either $\varphi \geq \omega_{1}$ or there are $\varphi_{n} \in \Phi(n<\omega)$ such that $\varphi \geq \varphi_{0}+\varphi_{1}+\cdots$.

PROOF. By Lemma 4.1 we may assume that $\varphi$ is right-irreducible. If $\varphi \nsupseteq \omega_{1}$, then the cofinality of $\varphi$ is $\omega$. Therefore, $\dot{\varphi}=\psi_{0}+\psi_{1}+\cdots$ for some types $\psi_{n}(n<\omega)$. The set $N=\left\{n: \psi_{n} \in \Phi\right\}$ is infinite since $\varphi$ is right-irreducible. If $N=\left\{n_{i}: i<\omega\right\}$, where $n_{0}<n_{1}<\cdots$, then $\varphi \geq \varphi_{0}+\varphi_{1}+\cdots$, where $\varphi_{i}=\psi_{n_{i}} \in \Phi$.

Suppose $(S,<)$ is an ordered set of type $\varphi \in \Phi$. We want to show that, if $[S]^{3}=K_{0} \cup K_{1}$, then either (3.1) or (3.2) holds.

The proof is essentially the same as above, the key step being to prove the analogue of Lemma 3.2. As before $A$ ( $A^{\prime}$ etc.) denotes a subset of $S$ of type $\omega$, but now $B\left(B^{\prime}\right.$ etc.) denotes a subset such that $\operatorname{tp}(B) \in \Phi$. There is one technical difficulty. We may clearly assume that $\varphi$ is such that $\left|\varphi^{\prime}\right|=|\varphi|$ whenever $\varphi^{\prime} \leq \varphi$ and $\varphi^{\prime} \in \Phi$. However, the cardinal $|\varphi|$ may be strictly greater than $\aleph_{1}$ (see $[\mathbf{1}]$ ). Because of this we have to replace the hypothesis $\mathrm{MA}_{\omega_{1}}$ by $\mathrm{MA}_{|\varphi|}$. With this modification, the proofs of Lemmas 3.1 and 3.2 are essentially as before. The only additional remark that is needed is that if $\varphi \geq \omega_{1}$, then $\varphi \geq \varphi_{0}+\varphi_{1}+\cdots$ where $\varphi_{i} \in \Phi$ by Lemma 4.2 , and this is enough to ensure that the sets $A_{n}^{\prime}(n<\omega)$ in the proof of Lemma 3.2 may be chosen so that $\bigcup_{n<\omega} A_{n}^{\prime}$ is not cofinal in $S$. This establishes (3.3) under a suitable hypothesis, but as before, by the remarks of Baumgartner and Hajnal [2], this extra hypothesis is inessential.

5. Concluding remarks. As we already stated, our method of proving (1.9) does not seem to yield (1.11), or even the weaker statements in (1.12). It should be noted that (1.11) is stronger than the Baumgartner-Hajnal theorem (1.10). In fact, by a standard argument due to Hajnal (see [4, Theorem 25.1]) if $\alpha$ is a limit ordinal and $n \rightarrow(3)_{k}^{2}$, then

$$
\varphi \rightarrow(\alpha, n)^{3}
$$

implies

$$
\varphi \rightarrow(\alpha)_{k}^{2} \text {. }
$$

For suppose that (5.1) holds and $S$ is an ordered set of type $\varphi$ and $[S]^{2}=\bigcup_{i<k} K_{i}$ is any $k$-partition of the pairs of $S$. Consider the partition $[S]^{3}=L_{0} \cup L_{1}$, wheie for $\alpha<\beta<\gamma$ we have $\{\alpha, \beta, \gamma\} \in L_{1}$ if and only if there are distinct $i, j<k$ such 
that $\{\alpha, \beta\} \in K_{i}$ and $\{\beta, \gamma\} \in K_{j}$. The fact that $n \rightarrow(3)_{k}^{2}$ implies that $n \notin\left[L_{1}\right]$. Therefore, by (5.1), there is a set $S^{\prime} \subseteq S$ such that $\operatorname{tp}\left(S^{\prime}\right)=\alpha$ and $\left[S^{\prime}\right]^{3} \subseteq L_{0}$. This implies that $\left[S^{\prime}\right]^{2} \subseteq K_{i}$ for some $i<k$ since $S^{\prime}$ has no last element.

Finally we point out that it is possible to extend Lemma 3.2 and replace $\omega+1$ by any $\alpha<\omega_{1}$. Using the same notation as in $\S 3$ we prove the following.

LEMMA 5.1. Assume $\mathrm{MA}_{\omega_{1}}$. Let $\alpha<\omega_{1}$ and let $B$ be any subset of $\omega_{1}$ such that (3.3) holds. Then there are sets $A_{\rho} \subseteq B(\rho<\alpha)$ such that $A_{0}<A_{1}<\cdots$ and $\left[A_{\rho}\right]^{2} \otimes\left[A_{\sigma}\right]^{1} \subseteq K_{1}(\rho<\sigma<\alpha)$.

Lemma 5.1 follows by an easy induction argument from Lemma 3.1 and the following lemma.

LEMMA 5.2. Assume $\mathrm{MA}_{\omega_{1}}$. Let $B \subseteq \omega_{1}$ and suppose that (3.3) holds. Let $A_{n}(n<\omega)$ be disjoint subsets of $B$. Then there are $A_{n}^{\prime} \subseteq A_{n}(n<\omega)$ and $B^{\prime} \subseteq B$ such that $\left[A_{n}^{\prime}\right]^{2} \otimes\left[B^{\prime}\right]^{1} \subseteq K_{1}(n<\omega)$.

PROOF. By Lemma 2.2 there is an ultrafilter $U_{n}$ on $A_{n}(n<\omega)$ such that, for each $\rho \in B$, there are $i(n, \rho)<2$ and a set $\tilde{A}_{n, \rho} \in \mathcal{U}_{n}$ such that $\left[\tilde{A}_{n, \rho}\right]^{2} \otimes[\{\rho\}]^{1} \subseteq$ $K_{i(n, \rho)}$. As in the proof of Lemma 3.2, we may assume that $i(n, \rho)=1$ for all $n<\omega$ and $\rho \in B$. By Lemma 2.1, there is $X_{n} \subseteq A_{n}(n<\omega)$ such that $X_{n} \backslash \tilde{A}_{n, \rho}$ is finite for all $\rho \in B$.

Let $P$ be the set of all ordered pairs $\langle n, s\rangle$ such that $n \in \omega, s \in[B]^{<\omega}$ and the set

$$
B(n, s)=\left\{\rho \in B:(\forall j \leq n)\left(X_{j} \backslash \tilde{A}_{j, \rho} \subseteq \bigcup_{\sigma \in s}\left(X_{j} \backslash \tilde{A}_{j, \sigma}\right)\right)\right\}
$$

is uncountable. It is easy to see that $P$ is not empty. Define a partial order $\leq_{P}$ on $P$ so that $\left\langle n^{\prime}, s^{\prime}\right\rangle \leq_{P}\langle n, s\rangle$ if and only if $n \leq n^{\prime}, s \subseteq s^{\prime}$ and $s^{\prime} \backslash s \subseteq B(n, s)$.

For $\langle n, s\rangle \in P$, let $l(\langle n, s\rangle)=\left\langle\bigcup_{\sigma \in s}\left(X_{j} \backslash \tilde{A}_{j, \sigma}\right): j \leq n\right\rangle$. Since there are only countably many different sequences $l(\langle n, s\rangle)$, it follows that, if $Q$ is any uncountable subset of $P$, then there are $n<\omega$ and distinct $\left\langle n, s_{1}\right\rangle,\left\langle n, s_{2}\right\rangle \in Q$ such that $l\left(\left\langle n, s_{1}\right\rangle\right)=l\left(\left\langle n, s_{2}\right\rangle\right)$. It follows that $\left\langle n, s_{1} \cup s_{2}\right\rangle$ is an element of $P$ which precedes both $\left\langle n, s_{1}\right\rangle$ and $\left\langle n, s_{2}\right\rangle$ in the order $\leq_{P}$. Thus $\left(P, \leq_{P}\right)$ satisfies the countable chain condition.

It is easy to check that the sets $\mathcal{E}_{k}=\{\langle n, s\rangle \in P: n \geq k\}(k<\omega)$ and $D_{\eta}=$ $\{\langle n, s\rangle \in P: s \backslash \eta \neq \varnothing\}\left(\eta<\omega_{1}\right)$ are dense in $\left(P, \leq_{P}\right)$, and so by $\mathrm{MA}_{\omega_{1}}$ there is a filter $\mp \subseteq P$ having nonempty intersection with all $\varepsilon_{k}(k<\omega)$ and $D_{\eta}\left(\eta<\omega_{1}\right)$. Put $B^{\prime}=\bigcup\{s:\langle n, s\rangle \in \mathcal{J}$ for some $n<\omega\}$.

For $k<\omega$ choose any $\langle n, s\rangle \in \mathcal{E}_{k} \cap \mathcal{F}$ and set

$$
A_{k}^{\prime}=X_{k} \cap \bigcap_{\sigma \in s} \tilde{A}_{k, \sigma}
$$

Notice that this definition of $A_{k}^{\prime}$ does not depend upon the particular choice of $\langle n, s\rangle$. For, if $\langle m, t\rangle \in \mathcal{E}_{k} \cap \mathcal{F}$, then there is $\langle l, r\rangle \in \mathcal{E}_{k} \cap \mathcal{F}$ which precedes both $\langle n, s\rangle$ and $\langle m, t\rangle$ in the order $\leq_{P}$ and this implies that

$$
X_{k} \cap \bigcap_{\sigma \in s} \tilde{A}_{k, \sigma}=X_{k} \cap \bigcap_{\sigma \in t} \tilde{A}_{k, \sigma}=X_{k} \cap \bigcap_{\sigma \in r} \tilde{A}_{k, \sigma}
$$

Now, for $k<\omega$ and $\rho \in B^{\prime}$ there is $\langle n, s\rangle \in \mathcal{F}$ such that $n \geq k$ and $\rho \in s$. Since $A_{k}^{\prime} \subseteq \tilde{A}_{k, \rho}$ and $i(k, \rho)=1$, it follows that $\left[A_{k}^{\prime}\right]^{2} \otimes\{\rho\} \subseteq K_{1}\left(k<\omega, \rho \in B^{\prime}\right)$. 


\section{REFERENCES}

1. J. Baumgartner, A new class of order types, Ann. of Math. Logic 9 (1976), 187-222.

2. J. Baumgartner and A. Hajnal, A proof (involving Martin's Axiom) of a partition relation, Fund. Math. 78 (1973), 193-203.

3. B. Dushnik and E. W. Miller, Partially ordered sets, Amer. J. Math. 63 (1941), 600-610.

4. P. Erdős, A. Hajnal, A. Máté and R. Rado, Combinatorial set theory, Akad. Kiadó, Budapest; North-Holland, Amsterdam, 1984.

5. P. Erdős and R. Rado, A partition calculus in set theory, Bull. Amer. Math. Soc. 62 (1956), 427-489.

6. F. Galvin, A letter addressed to P. Erdős dated October 12, 1970.

7. , On a partition theorem of Baumgartner and Hajnal, Colloq. Math. Soc. Janos Bolyai 10 (1973), 711-729.

8. T. Jech, Set theory, Academic Press, New York, 1978.

9. M. E. Rudin, Martin's Axiom, Handbook of Mathematical Logic (J. Barwise, Ed.), NorthHolland, Amsterdam, 1977, pp. 491-501. CANADA

Department of Mathematics, University of Minnesota, Minneapolis, MinNESOTA 55455 